\title{
Performance Analysis and Optimization of Cache-Enabled Small Cell Networks
}

\author{
Yongxu Zhu*, Gan Zheng*, Lifeng Wang ${ }^{\dagger}$, Kai-Kit Wong ${ }^{\dagger}$, and Liqiang Zhao ${ }^{\ddagger}$ \\ ${ }^{*}$ Wolfson School of Mechanical, Electrical and Manufacturing Engineering, Loughborough University, UK \\ ${ }^{\dagger}$ Department of Electronic and Electrical Engineering, University College London, London, UK \\ $¥$ Xidian University, Xian, China
}

\begin{abstract}
This paper studies the performance of cache-enabled dense small cell networks consisting of multi-antenna sub-6 GHz and millimeter-wave base stations. We first derive the successful content delivery probability by accounting for the key channel features at sub-6 GHz and mmWave frequencies. In general, the optimal content placement is unknown when the base stations have multiple antennas. Then we propose a simple yet effective probabilistic content placement scheme to maximize the successful content delivery probability, which could balance caching both the most popular contents and achieving content diversity. Numerical results demonstrate that our proposed content placement scheme yields significantly better performance than only caching the most popular contents. The comparisons between the sub-6 GHz and millimeter-wave systems reveal an interesting tradeoff between caching capacity and base station density for the millimeter-wave system to achieve similar performance as the sub-6 GHz system.
\end{abstract}

\section{INTRODUCTION}

To meet the high capacity requirement for the future mobile networks, one promising solution is to deploy dense small cell base stations (SBSs) in the existing macrocell cellular networks. While more small cells shortens the communication distance, the major challenge is to transfer huge amount of mobile data from the core networks to the small cells and this imposes stringent demands on backhaul links. To address this problem, caching popular contents at small cells has been proposed as one of the most effective solutions, considering the fact that most mobile data are contents such as video, weather forecasts, news and maps, that are repeatedly requested and cacheable [1]. Cache-enabled small cells will bring content closer to users, decrease backhaul traffic and reduce transmission delays, thus alleviating many bottleneck problems in wireless content delivery networks. This paper focuses on the caching design at both sub- $6 \mathrm{GHz}$, and millimeter-wave (mmWave).

MmWave communication has received much interest for providing high capacity because there are vast amount of inexpensive spectra available in $30 \mathrm{GHz}$ to $300 \mathrm{GHz}$. However, compared to sub- $6 \mathrm{GHz}$ frequencies, mmWave channel experiences excessive attenuation due to rainfall, atmospheric or gaseous absorption, and is susceptible to blockage. To achieve similar performance as the sub- $6 \mathrm{GHz}$ systems, more expensive resources are required in mmWave systems, such as antennas [2] and high density deployment of SBSs [3]. In this regard, caching can be a cost-effective alternative to boost the performance of mmWave communications. Cache assignment with video streaming in mmWave SBSs on the highway is discussed in [4] and it is shown to significantly reduce the connection and retrieval delays.

Content placement with limited cache size is the key issue in caching design, since unplanned caching in nearby SBSs will result in more interference. The traditional method of caching most popular content (MPC) in wired networks is no longer optimal when considering the wireless transmission. A strategy that combines MPC and the largest content diversity caching is proposed in [5], together with cooperative transmission in cluster-centric small cell networks. This strategy is extended to the distributed relay networks with relay clustering in [6] to combat the half-duplex constraint, and it significantly improves the outage performance. Caching distribution in device-to-device (D2D) communication networks is studied in [7] under a simple transmission strategy where a single file is transmitted at a time. Several spatially correlated caching strategies are investigated for D2D networks in [8] in contrast to the baseline independent content placement.

The existing research for caching design in SBSs are restricted to the single-antenna case and mainly for the sub$6 \mathrm{GHz}$ band. Little is known about the impact of multiple antennas at the densely deployed cache-enabled SBSs and the adoption of mmWave band. In this paper, we analyze the performance of caching in multi-antenna SBSs in sub-6 GHz and mmWave networks. We derive successful content delivery probability (SCDP) of multi-antenna SBSs, and propose a heuristic algorithm to maximize the SCDP via probabilistic content placement. The numerical results show that in contrast to the traditional way of deploying much higher density SBSs or installing many more antennnas, increasing caching capacity at mmWave SBSs provides a low-cost solution to achieve comparable SCDP performance as sub-6 GHz systems.

\section{SySTEM MODEL}

We consider a cache-enabled dense small cell networks consisting of the sub- $6 \mathrm{GHz}$ and mmWave SBSs tiers. In such networks, each user equipment (UE) in one tier is associated with the nearest SBS that has cached the desired content. We assume that there are a finite content library denoted as $\mathcal{F}:=\left\{f_{1}, \ldots, f_{j}, \ldots, f_{J}\right\}$, where $f_{j}$ is the $j$-th most popular content and the number of contents is $J$. Each content has the same size and each BS can only store up to $M$ contents. It is assumed that $M \ll J$. The content request 
probability is modeled as the Zipf distribution and is given by $a_{j}=j^{-\gamma} / \sum_{m=1}^{J} m^{-\gamma}$, where $\gamma$ is the Zipf parameter.

\section{A. Probabilistic Content Placement}

We consider a probabilistic caching model where the content is independently stored with the same probability in all SBSs of the same tier (either sub-6 GHz or mmWave) [9]. Let $b_{j}$ denote the probability that the $j$-th content is cached at a SBS. In the probabilistic caching strategy, the caching probability $\left\{b_{j}\right\}$ needs to satisfy $\sum_{j=1}^{J} b_{j} \leqslant M$, where $0 \leqslant b_{j} \leqslant 1, \forall j$.

\section{B. Downlink Transmission}

In the considered downlink networks, each sub-6 GHz SBS is equipped with $N_{\mathrm{s}}$ antennas, and each mmWave SBS has directional mmWave antennas. All UEs are single-antenna nodes. The positions of sub- $6 \mathrm{GHz}$ SBSs are modeled by a homogeneous Poisson point process (HPPP) $\Phi^{\mathrm{S}}$ with the density $\lambda_{\mathrm{s}}$, and the positions of mmWave SBSs are modeled by an independent HPPP $\Phi^{\mathrm{mm}}$ with the density $\lambda_{\mathrm{mm}}$. Define $\Phi_{j}^{\mathrm{s}}$ and $\Phi_{j}^{\mathrm{mm}}$ as the point process corresponding to all SBSs that cache the content $j$ in the sub- $6 \mathrm{GHz}$ tier and the mmWave tier with the density $b_{j} \lambda_{\mathrm{s}}$ and $b_{j} \lambda_{\mathrm{mm}}$.

1) sub-6 GHz Tier: In the sub-6 GHz tier, the maximumratio transmission beamforming is adopted at each SBS. All channels undergo independent identically distributed (i.i.d.) quasi-static Rayleigh block fading. Without loss of generality, when a typical sub- $6 \mathrm{GHz}$ UE located at the origin $o$ requests the content $j$ from the associated sub- $6 \mathrm{GHz}$ BS $X_{o}$ that has cached this content, its received signal-to-interference-plusnoise ratio (SINR) is given by

$$
\mathrm{SINR}_{j}^{\mathrm{s}}=\frac{P_{\mathrm{s}} h_{j}^{\mathrm{s}} L\left(\left|X_{j}^{\mathrm{s}}\right|\right)}{\mathcal{I}_{j}^{\mathrm{s}}+\overline{\mathcal{I}}_{j}^{\mathrm{s}}+\sigma_{\mathrm{s}}^{2}},
$$

where $P_{\mathrm{s}}$ is the transmit power, $h_{j}^{\mathrm{s}} \sim \Gamma\left(N_{\mathrm{s}}, 1\right)$ is the channel power gain between the typical sub- $6 \mathrm{GHz} \mathrm{UE}$ and its serving sub-6 GHz SBS and $\Gamma(\cdot, \cdot)$ denotes Gamma distribution. The path loss is $L\left(\left|X_{j}^{\mathrm{s}}\right|\right)=\beta_{\mathrm{s}}\left(\left|X_{j}^{\mathrm{s}}\right|\right)^{-\alpha_{\mathrm{s}}}$ with the distance $\left|X_{j}^{\mathrm{s}}\right|$, where $\beta_{\mathrm{s}}$ is the frequency dependent constant parameter and $\alpha_{\mathrm{s}}$ is the path loss exponent. $\sigma_{\mathrm{s}}^{2}$ is the noise power at a sub$6 \mathrm{GHz}$ UE. The inter-cell interference are given by $\mathcal{I}_{j}^{\mathrm{s}}=$ $\sum_{i \in \Phi_{j}^{\mathrm{s}} \backslash X_{o}} P_{\mathrm{s}} h_{i, o} L\left(\left|X_{i, o}\right|\right)$ and $\overline{\mathcal{I}}_{j}^{\mathrm{s}}=\sum_{k \in \bar{\Phi}_{j}^{\mathrm{s}}} P_{\mathrm{s}} h_{k, o} L\left(\left|X_{k, o}\right|\right)$, where $\Phi_{j}^{\mathrm{s}} \backslash X_{o}$ is the point process with density $b_{j} \lambda_{\mathrm{s}}$ corresponding to the interfering SBSs that cache the content $j$, and $\bar{\Phi}_{j}^{\mathrm{s}}=\Phi^{\mathrm{s}}-\Phi_{j}^{\mathrm{s}}$ with density $\left(1-b_{j}\right) \lambda_{\mathrm{s}}$ is the point process corresponding to the interfering SBSs that do not store the content $j . h_{i, o}, h_{k, o} \sim \exp (1)$ are the interfering channel power gains that follow the exponential distribution.

2) mmWave Tier: In the mmWave tier, we assume that the directional beamforming is adopted at each mmWave BS and the small-scale fading is neglected, as verified by the practical channel measurements such as [10]. Unlike the conventional sub-6 GHz counterpart, mmWave transmissions are highly sensitive to the blockage. According to the average line-ofsight (LOS) model in [11-13], we consider that the mmWave link is LOS if the communication distance is less than $D_{L}$, and otherwise it is non-line-of-sight (NLOS). Moreover, the existing literature has confirmed that mmWave transmissions tend to be noise-limited and interference is weak $[11,13]$. Therefore, when a typical mmWave UE requests the content $j$ from the associated mmWave SBS that has cached this content, its received SINR is given by

$$
\mathrm{SINR}_{j}^{\mathrm{mm}}=\frac{P_{\mathrm{mm}} G_{\mathrm{mm}} L\left(\left|Y_{j}^{\mathrm{mm}}\right|\right)}{\sigma_{\mathrm{mm}}^{2}},
$$

where $P_{\mathrm{mm}}$ is the transmit power of the mmWave SBS, $G_{\mathrm{mm}}$ is the main-lobe gain of using direction beamforming and equal to the number of antenna elements [14]. The path loss is expressed as $L\left(\left|Y_{j}^{\mathrm{mm}}\right|\right)=\beta_{\mathrm{mm}}\left(\left|Y_{j}^{\mathrm{mm}}\right|\right)^{-\alpha}$ with the distance $\left|Y_{j}^{\mathrm{mm}}\right|$ and frequency-dependent parameter $\beta_{\mathrm{mm}}$. The path loss exponent $\alpha=\alpha_{\mathrm{L}}$ when it is a LOS link and $\alpha=\alpha_{\mathrm{N}}$ when it is an NLOS link. $\sigma_{\mathrm{mm}}^{2}$ is the combined power of noise and weak interference.

\section{Successful Content Delivery Probability}

In this paper, SCDP is used as the performance indicator, which represents the probability that a content requested by a typical UE is both cached in the network and can be successfully transmitted to the UE. Assuming that each content has $\eta$ bits, which needs to be delivered during the time $T$. By using the law of total probability, the SCDP in the sub- $6 \mathrm{GHz}$ tier is calculated as

$$
\mathcal{P}_{\mathrm{SCD}}^{\mathrm{s}}=\sum_{j=1}^{J} a_{j} \operatorname{Pr}\left(\mathrm{SINR}_{\mathrm{j}}^{\mathrm{s}}>\varphi_{\mathrm{s}}\right)
$$

where $\varphi_{\mathrm{s}}=2^{\frac{\eta}{W_{\mathrm{s}} T}}-1, W_{\mathrm{s}}$ is the sub-6 $\mathrm{GHz}$ bandwidth. Likewise, in the mmWave tier, the SCDP is calculated as

$$
\mathcal{P}_{\mathrm{SCD}}^{\mathrm{mm}}=\sum_{j=1}^{J} a_{j} \operatorname{Pr}\left(\mathrm{SINR}_{\mathrm{j}}^{\mathrm{mm}}>\varphi_{\mathrm{mm}}\right),
$$

where $\varphi_{\mathrm{mm}}=2^{\frac{\eta}{W_{\mathrm{mm} T}}}-1$, and $W_{\mathrm{mm}}$ denotes the mmWave bandwidth. The rest of this section is devoted to deriving the SCDPs in (3) and (4).

\section{A. sub-6 GHz Tier}

Based on (1) and (3), the SCDP in the sub-6 GHz tier can be derived and summarized below.

Theorem 1: In the cache-enabled sub-6 GHz tier, the SCDP is given by

$$
\mathcal{P}_{\mathrm{SCD}}^{\mathrm{s}}=\sum_{j=1}^{J} a_{j} \mathcal{P}_{j, \mathrm{SCD}}^{\mathrm{s}}\left(b_{j}\right),
$$

where $P_{j, \mathrm{SCD}}^{\mathrm{s}}\left(b_{j}\right)$ denotes the probability that the $j$-th request content is successfully delivered to the sub- $6 \mathrm{GHz}$ UE by its serving SBS, and is expressed as

$$
P_{j, \mathrm{SCD}}^{\mathrm{s}}\left(b_{j}\right)=\int_{0}^{\infty} P_{\mathrm{cov}}^{\mathrm{s}}\left(x, b_{j}\right) f_{\left|X_{j}^{\mathrm{s}}\right|}(x) d x,
$$

where $P_{\mathrm{cov}}^{\mathrm{s}}\left(x, b_{j}\right)$ is given by $(7)$, which represents the conditional coverage probability that the received SINR is larger than $\varphi_{\mathrm{s}}$ given a typical communication distance $x . f_{\left|X_{j}^{\mathrm{s}}\right|}(x)$ is the probability density function (PDF) of the distance $\left|X_{j}^{\mathrm{s}}\right|$ between a typical sub- $6 \mathrm{GHz} \mathrm{UE}$ and its nearest serving 


$$
\begin{aligned}
& P_{\mathrm{cov}}^{\mathrm{s}}\left(x, b_{j}\right)=\sum_{n=0}^{N_{\mathrm{s}}-1} \frac{\left(x^{\alpha_{\mathrm{s}}}\right)^{n}}{n !(-1)^{n}} \sum_{\left\{t_{q}\right\}_{q=1}^{n} \in \Theta} \frac{n !}{\prod_{q=1}^{n} t_{q} !(q !)^{t_{q}}} \exp \left(-\frac{\varphi_{\mathrm{s}} \sigma_{\mathrm{s}}^{2} x^{\alpha_{\mathrm{s}}}}{P_{\mathrm{s}} \beta_{\mathrm{s}}}-2 \pi b_{j} \lambda_{\mathrm{s}} \frac{\varphi_{\mathrm{s}} x^{2}}{\alpha_{\mathrm{s}}-2}\right. \\
& \left.{ }_{2} F_{1}\left[1, \frac{-2+\alpha_{\mathrm{s}}}{\alpha_{\mathrm{s}}}, 2-\frac{2}{\alpha_{\mathrm{s}}},-\varphi_{\mathrm{s}}\right]-\frac{2 \pi^{2}}{\alpha_{\mathrm{s}}}\left(1-b_{j}\right) \lambda_{\mathrm{s}}\left(\varphi_{\mathrm{s}} x^{\alpha_{\mathrm{s}}}\right)^{\frac{2}{\alpha_{\mathrm{s}}}} \csc \left(\frac{2 \pi}{\alpha_{\mathrm{s}}}\right)\right) \prod_{q=1}^{n}\left(\mathcal{T}^{(q)}\left(x^{\alpha_{\mathrm{s}}}\right)\right)^{t_{q}},
\end{aligned}
$$

where $\Theta \triangleq\left\{\left\{t_{q}\right\}_{q=1}^{n} \mid \sum_{q=1}^{n} q \cdot t_{q}=n, t_{q}\right.$ is an integer, $\left.\forall n\right\}, \csc (\cdot)$ is the Cosecant trigonometry function, and

$$
\begin{aligned}
\mathcal{T}_{j}^{(1)}\left(x^{\alpha_{\mathrm{s}}}\right)= & -\frac{\varphi_{\mathrm{s}} \sigma_{\mathrm{s}}^{2}}{P_{\mathrm{s}} \beta}-2 \pi b_{j} \lambda_{\mathrm{s}} x^{2-\alpha_{\mathrm{s}}} \varphi_{\mathrm{s}} \frac{\alpha_{\mathrm{s}}-2+2\left(1+\varphi_{\mathrm{s}}\right){ }_{2} F_{1}\left[1, \frac{\alpha_{\mathrm{s}}-2}{\alpha_{\mathrm{s}}}, 2-\frac{2}{\alpha_{\mathrm{s}}},-\varphi_{\mathrm{s}}\right]}{\left(1+\varphi_{\mathrm{s}}\right)\left(\alpha_{\mathrm{s}}-1\right) \alpha_{\mathrm{s}}} \\
& -4 \pi^{2}\left(1-b_{j}\right) \lambda_{\mathrm{s}}\left(\varphi_{\mathrm{s}}^{\alpha_{\mathrm{s}}} x\right)^{2-\alpha_{\mathrm{s}}} \csc \left(\frac{2 \pi}{\alpha_{\mathrm{s}}}\right), \\
\mathcal{T}_{j}^{(q)}\left(x^{\alpha_{\mathrm{s}}}\right)= & 2 \pi b_{j} \lambda_{\mathrm{s}} q !(-1)^{q} x^{-\left(2+\alpha_{\mathrm{s}}\right)(1+q)} \varphi_{\mathrm{s}}^{-q(1+q)} \frac{{ }_{2} F_{1}\left[1+q, \frac{2+\alpha_{\mathrm{s}}}{\alpha_{\mathrm{s}}}, 2+\frac{2}{\alpha_{\mathrm{s}}},-\frac{1}{\varphi_{\mathrm{s}}}\right]}{2+\alpha_{\mathrm{s}}}+2 \pi\left(1-b_{j}\right) \lambda_{\mathrm{s}} q !(-1)^{q} \\
& \times\left(x^{\alpha_{\mathrm{s}}}\right)^{-q+\frac{2}{\alpha_{\mathrm{s}}}} \varphi_{\mathrm{s}}^{\frac{2}{\alpha_{\mathrm{s}}}} \frac{\Gamma\left(q-\frac{2}{\alpha_{\mathrm{s}}}\right) \Gamma\left(\frac{2+\alpha_{\mathrm{s}}}{\alpha_{\mathrm{s}}}\right)}{\alpha_{\mathrm{s}} \Gamma(1+q)}, q>1 .
\end{aligned}
$$

SBS that stores content $j$, which is given by $f_{\left|X_{j}^{\mathrm{s}}\right|}(x)=$ $2 \pi b_{j} \lambda_{\mathrm{s}} x e^{-\pi b_{j} \lambda_{\mathrm{s}} x^{2}}$.

Proof 1: Based on (3), $\mathcal{P}_{\mathrm{SCD}}^{\mathrm{s}}$ is calculated as

$$
\mathcal{P}_{\mathrm{SCD}}^{\mathrm{s}}=\sum_{j=1}^{J} a_{j} \int_{0}^{\infty} \underbrace{\operatorname{Pr}\left(\operatorname{SINR}_{\mathrm{j}}^{\mathrm{s}}(x)>\varphi_{\mathrm{s}}\right)}_{P_{\mathrm{cov}}^{\mathrm{s}}\left(x, b_{j}\right)} f_{\left|X_{j}^{\mathrm{s}}\right|}(x) d x
$$

where $P_{\mathrm{cov}}^{\mathrm{s}}\left(x, b_{j}\right)$ is the conditional coverage probability, and $f_{\left|X_{j}^{\mathrm{s}}\right|}(x)$ is the PDF of the distance $\left|X_{j}^{\mathrm{s}}\right|$. Then, we derive $P_{\mathrm{cov}}^{\mathrm{s}}\left(x, b_{j}\right)$ as

$$
\begin{aligned}
& P_{\mathrm{cov}}^{\mathrm{s}}\left(x, b_{j}\right)=\int_{0}^{\infty} \operatorname{Pr}\left(h_{j}^{\mathrm{s}}>s\left(\tau+\sigma_{\mathrm{s}}^{2}\right)\right) d \operatorname{Pr}\left(\hat{\mathcal{I}}_{j}^{\mathrm{s}} \leqslant \tau\right) \\
& =\int_{0}^{\infty} e^{-s\left(\tau+\sigma_{\mathrm{s}}^{2}\right)} \sum_{n=0}^{N_{\mathrm{s}}-1} \frac{\left(s\left(\tau+\sigma_{\mathrm{s}}^{2}\right)\right)^{n}}{n !} d \operatorname{Pr}\left(\hat{\mathcal{I}}_{j}^{\mathrm{s}} \leqslant \tau\right)
\end{aligned}
$$

where $\hat{\mathcal{I}}_{j}^{\mathrm{s}}=\mathcal{I}_{j}^{\mathrm{s}}+\overline{\mathcal{I}}_{j}^{\mathrm{s}}$ and $s=\frac{\varphi_{\mathrm{s}} x^{\alpha_{\mathrm{s}}}}{P_{\mathrm{s}} \beta_{\mathrm{s}}}$. Note that

$$
\begin{aligned}
& \left.\frac{d^{n}\left(\exp \left(-s\left(\tau+\sigma_{\mathrm{s}}^{2}\right)\right)\right)}{d \nu^{n}}\right|_{\nu=x^{\alpha_{\mathrm{s}}}} \\
& =\left(-\frac{s\left(\tau+\sigma_{\mathrm{s}}^{2}\right)}{\nu}\right)^{n} \exp \left(-s\left(\tau+\sigma_{\mathrm{s}}^{2}\right)\right) .
\end{aligned}
$$

By using (12), (11) can be rewritten as

$$
\begin{aligned}
P_{\mathrm{cov}}^{\mathrm{s}}\left(x, b_{j}\right) & =\sum_{n=0}^{N_{\mathrm{s}}-1} \frac{x^{n \alpha_{\mathrm{s}}}}{n !(-1)^{n}} \\
& \times\left.\frac{d^{n}\left[\exp \left(-s \sigma_{\mathrm{s}}^{2}\right) \mathcal{L}_{\mathcal{I}_{j}^{\mathrm{s}}}(s) \mathcal{L}_{\overline{\mathcal{I}}_{j}^{\mathrm{s}}}(s)\right]}{d \nu^{n}}\right|_{\nu=x^{\alpha_{\mathrm{s}}}},
\end{aligned}
$$

where $\mathcal{L}_{\mathcal{I}_{j}^{\mathrm{s}}}(\cdot)$ is the Laplace transform of the PDF $\mathcal{I}_{j}^{\mathrm{s}}$, and $\mathcal{L}_{\overline{\mathcal{I}}_{j}^{\mathrm{s}}}(\cdot)$ is the Laplace transform of the PDF $\overline{\mathcal{I}}_{j}^{\mathrm{s}}$. Then $\mathcal{L}_{\mathcal{I}_{j}^{\mathrm{s}}}(s)$ is given by

$$
\begin{aligned}
& \mathcal{L}_{\mathcal{I}_{j}^{\mathrm{s}}}(s)=\mathbb{E}_{\Phi_{j}^{\mathrm{s}}}\left[\exp \left(-s \sum_{i \in \Phi_{j}^{\mathrm{s}} \backslash \mathrm{o}} P_{\mathrm{s}} h_{i, o} L\left(\left|X_{i, o}\right|\right)\right)\right] \\
& =\exp \left[-\int_{x}^{\infty}\left(1-\mathbb{E}_{h_{i, o}}\left\{\exp \left(-s P_{\mathrm{s}} h_{i, o} \beta_{\mathrm{s}} r^{-\alpha_{\mathrm{s}}}\right)\right\}\right) 2 \pi b_{j} \lambda_{\mathrm{s}} r d r\right] .
\end{aligned}
$$

Likewise, $\mathcal{L}_{\overline{\mathcal{I}}_{j}^{\mathrm{s}}}(s)$ is given by

$$
\begin{aligned}
\mathcal{L}_{\overline{\mathcal{I}}_{j}^{\mathrm{s}}}(s)= & \mathbb{E}_{\overline{\mathcal{I}}_{j}^{\mathrm{s}}}\left[\exp \left(-s \sum_{k \in \bar{\Phi}_{j}^{\mathrm{s}}} P_{\mathrm{s}} h_{k, o} L\left(\left|X_{k, o}\right|\right)\right)\right] \\
=\exp [ & -\int_{0}^{\infty}\left(1-\mathbb{E}_{h_{k, o}}\left\{\exp \left(-s P_{\mathrm{s}} h_{k, o} \beta_{\mathrm{s}} r^{-\alpha_{\mathrm{s}}}\right)\right\}\right) \\
& \left.\times 2 \pi\left(1-b_{j}\right) \lambda_{\mathrm{s}} r d r\right]
\end{aligned}
$$

Substituting (14) and (15) into (13) and after some manipulations, we can obtain the desired result (7).

Note that $P_{j, \mathrm{SCD}}^{\mathrm{s}}\left(b_{j}\right)$ becomes the probability of successful transmission from the serving SBS to the typical user when $b_{j}=1$ in traditional sub- $6 \mathrm{GHz}$ networks without caching.

\section{B. mmWave Tier}

Based on (2) and (4), the SCDP in the mmWave Tier can be derived and summarized below.

Theorem 2: In the cache-enabled mmWave tier, the SCDP is given by

$$
\mathcal{P}_{\mathrm{SCD}}^{\mathrm{mm}}=\sum_{j=1}^{J} a_{j} \mathcal{P}_{j, \mathrm{SCD}}^{\mathrm{mm}, \mathrm{L}}\left(b_{j}\right)+\sum_{j=1}^{J} a_{j} \mathcal{P}_{j, \mathrm{SCD}}^{\mathrm{mm}, \mathrm{N}}\left(b_{j}\right),
$$


where $\mathcal{P}_{j, \mathrm{SCD}}^{\mathrm{mm}, \mathrm{L}}\left(b_{j}\right)$ and $\mathcal{P}_{j, \mathrm{SCD}}^{\mathrm{mm}, \mathrm{N}}\left(b_{j}\right)$ denote that probabilities that the content $j$ is successfully delivered when the mmWave UE is connected to its serving mmWave SBS via LOS link and NLOS link, and are given by

$$
\begin{gathered}
\mathcal{P}_{j, \mathrm{SCD}}^{\mathrm{mm}, \mathrm{L}}\left(b_{j}\right)=1-e^{-\left(\min \left(D_{L}, d_{\mathrm{L}}\right)\right)^{2} \pi b_{j} \lambda_{\mathrm{mm}}}, \\
\mathcal{P}_{j, \mathrm{SCD}}^{\mathrm{mm}, \mathrm{N}}\left(b_{j}\right)=e^{-D_{L}^{2} \pi b_{j} \lambda_{\mathrm{mm}}}-e^{-\left(\max \left(D_{L}, d_{\mathrm{N}}\right)\right)^{2} \pi b_{j} \lambda_{\mathrm{mm}}},
\end{gathered}
$$

respectively, where $d_{\mathrm{L}}=\left(\frac{P_{\mathrm{mm}} G_{\mathrm{mm}} \beta_{\mathrm{mm}}}{\varphi_{\mathrm{mm}} \sigma_{\mathrm{mm}}^{2}}\right)^{\frac{1}{\alpha_{\mathrm{L}}}}$ and $d_{\mathrm{N}}=$ $\left(\frac{P_{\mathrm{mm}} G_{\mathrm{mm}} \beta_{\mathrm{mm}}}{\varphi_{\mathrm{mm}} \sigma_{\mathrm{mm}}^{2}}\right)^{\frac{1}{\alpha_{\mathrm{N}}}}$.

Proof 2: Based on (2) and (4), the SCDP for a LOS mmWave link can be derived as

$$
\begin{aligned}
& \mathcal{P}_{\mathrm{SCD}}^{\mathrm{mm}, \mathrm{L}}=\int_{0}^{D_{L}} \operatorname{Pr}\left(\frac{P_{\mathrm{mm}} G_{\mathrm{mm}} \beta_{\mathrm{mm}} y^{-\alpha_{\mathrm{L}}}}{\sigma_{\mathrm{mm}}^{2}}>\varphi_{\mathrm{mm}}\right) f_{\left|Y_{j}^{\mathrm{mm}}\right|}(y) d y \\
& \quad=\mathbb{1}\left(D_{L}<d_{\mathrm{L}}\right) \int_{0}^{D_{L}} f_{\left|Y_{j}^{\mathrm{mm}}\right|}(y) d y \\
& \quad+\mathbb{1}\left(D_{L}>d_{\mathrm{L}}\right) \int_{0}^{d_{\mathrm{L}}} f_{\left|Y_{j}^{\mathrm{mm}}\right|}(y) d y
\end{aligned}
$$

where $f_{\left|Y_{j}^{\mathrm{mm} \mid}\right|}(y)$ is the PDF of the distance $\left|Y_{j}^{\mathrm{mm}}\right|$ between a typical user and its serving mmWave SBS, which is given by $f_{\left|Y_{j}^{\mathrm{mm}}\right|}(y)=2 \pi b_{j} \lambda_{\mathrm{mm}} y e^{-\pi b_{j} \lambda_{\mathrm{mm}} y^{2}}$ and $y \geqslant 0$. Similarly, the SCDP for a NLOS mmWave link can be derived as

$$
\begin{aligned}
& \mathcal{P}_{\mathrm{SCD}}^{\mathrm{mm}, \mathrm{N}}=\int_{D_{L}}^{\infty} \operatorname{Pr}\left(\frac{P_{\mathrm{mm}} G_{\mathrm{mm}} \beta_{\mathrm{mm}} y^{-\alpha_{\mathrm{N}}}}{\sigma_{\mathrm{mm}}^{2}}>\varphi_{\mathrm{mm}}\right) f_{\left|Y_{j}^{\mathrm{mm}}\right|}(y) d y \\
& =\mathbb{1}\left(D_{L}<d_{\mathrm{N}}\right) \int_{D_{L}}^{d_{\mathrm{N}}} f_{\left|Y_{j}^{\mathrm{mm}}\right|}(y) d y .
\end{aligned}
$$

Thus, we obtain the SCDP expressions (17) and (18) for a LOS/NLOS mmWave link.

\section{Optimization of Probabilistic Content PLACEMENT}

In this section, we seek to maximize the SCDP by optimizing the probabilistic content placement $\left\{b_{j}\right\}$. The main difficulty is that the SCDP expressions (5) and (16) do not have a closed-form solution for the multi-antenna case, which is much more challenging than the single-antenna SBS case studied in [15]. To tackle this problem, here we propose a heuristic algorithm based on the combination of MPC and CD content placement scheme. In the proposed scheme, a fraction of caching space $\varepsilon M(0 \leqslant \varepsilon \leqslant 1)$ at a SBS is allocated to store the most popular contents which is called the MPC region. The remaining cache space is allocated to randomly store the contents with certain probabilities and is called the CD region.

In this scheme, the content placement probabilities $\left\{b_{j}\right\}$ need to satisfy the following conditions:

$$
b_{j}=\left\{\begin{array}{l}
1, j \in[1, \ldots,\lfloor\varepsilon M\rfloor] \\
\varpi, j \in[\lfloor\varepsilon M\rfloor+1, \ldots,\lfloor\varepsilon M\rfloor+\lfloor(1-\varepsilon) M / \varpi\rfloor] \\
0, j \in[\lfloor\varepsilon M\rfloor+\lfloor(M-\varepsilon M) / \varpi\rfloor+1, \ldots, J]
\end{array}\right.
$$

which are characterized by two variables $\varepsilon$ and $\varpi$, where $\varpi$ denotes the common probability value that a content in the $\mathrm{CD}$ region is stored at a SBS.

As such, the SCDP of the sub-6 GHz tier or mmWave tier can be expressed as

$$
\mathcal{P}_{\mathrm{SCD}}^{\mathrm{s}, \mathrm{mm}}=\sum_{j=1}^{\lfloor\varepsilon M\rfloor} a_{j} \mathcal{P}_{j, \mathrm{SCD}}^{\mathrm{s}, \mathrm{mm}}(1)+\sum_{j=\lfloor\varepsilon M\rfloor+1}^{\lfloor\varepsilon M\rfloor+\left\lfloor\frac{M-\lfloor\varepsilon M\rfloor}{\varpi}\right\rfloor} a_{j} \mathcal{P}_{j, \mathrm{SCD}}^{\mathrm{s}, \mathrm{mm}}(\varpi)
$$

It is seen in (22) that contents $\{1, \cdots,\lfloor\varepsilon M\rfloor\}$ have the same SCDP $\mathcal{P}_{j, \mathrm{SCD}}^{\mathrm{s}, \mathrm{mm}}(1)$, and contents $\left\{\lfloor\varepsilon M\rfloor+1, \cdots,\lfloor\varepsilon M\rfloor+\left\lfloor\frac{M-\lfloor\varepsilon M\rfloor}{\varpi}\right\rfloor\right\}$ have the same SCDP $\mathcal{P}_{j, \mathrm{SCD}}^{\mathrm{s}, \mathrm{mm}}(\varpi)$. Our aim is to maximize the overall SCDP, and the problem is formulated as

$$
\begin{array}{ll}
\max _{\varepsilon, \varpi} & \mathcal{P}_{\mathrm{SCD}}^{\mathrm{s}, \mathrm{mm}} \text { in }(22) \\
\text { s.t. } & \mathrm{C} 1: 0 \leqslant \varepsilon \leqslant 1, \\
& \mathrm{C} 2: 0 \leqslant \varpi \leqslant 1, \\
& \mathrm{C} 3: \mathbf{1}(\varepsilon=1) \varpi=0,
\end{array}
$$

where $\mathbf{1}(A)$ is the indicator function that returns one if the condition A is satisfied. The problem (23) is non-convex, and finding its global optimal solution is challenging. To obtain an efficient caching placement solution, we first use the following approximations [16]

$$
\left\{\begin{array}{l}
\sum_{j=1}^{\lfloor\varepsilon M\rfloor} a_{j} \approx \frac{(\varepsilon M)^{1-\gamma}-1}{J^{1-\gamma}-1} \\
\sum_{j=\lfloor\varepsilon M\rfloor+1}^{\lfloor\varepsilon M\rfloor+\left\lfloor\frac{M-\lfloor\varepsilon M\rfloor}{\varpi}\right\rfloor} a_{j} \approx \frac{M^{1-\gamma}}{J^{1-\gamma}-1}\left[\left(\varepsilon+\frac{(1-\varepsilon)}{\varpi}\right)^{1-\gamma}-\varepsilon^{1-\gamma}\right]
\end{array}\right.
$$

respectively, based on the fact that for Zipf popularity with $0<\gamma, \gamma \neq 1$ and large $J(M \ll J)$, we have $\sum_{j=1}^{M} j^{-\gamma} / \sum_{m=1}^{J} m^{-\gamma} \approx\left(M^{1-\gamma}-1\right) /\left(J^{1-\gamma}-1\right)$ [16]. Therefore, the objective function of (22) can be approximated as

$$
\begin{aligned}
& \widetilde{\mathcal{P}}_{\mathrm{SCD}}^{\mathrm{s}, \mathrm{mm}} \approx \mathcal{P}_{j, \mathrm{SCD}}^{\mathrm{s}, \mathrm{mm}}(\varpi) \frac{M^{1-\gamma}}{J^{1-\gamma}-1}\left[\left(\varepsilon+\frac{(1-\varepsilon)}{\varpi}\right)^{1-\gamma}-\varepsilon^{1-\gamma}\right] \\
& +\mathcal{P}_{j, \mathrm{SCD}}^{\mathrm{s}, \mathrm{mm}}(1) \frac{M^{1-\gamma}}{J^{1-\gamma}-1} \varepsilon^{1-\gamma}-\frac{\mathcal{P}_{j, \mathrm{SCD}}^{\mathrm{s}}(1)}{J^{1-\gamma}-1} .
\end{aligned}
$$

Note that for the special case of MPC caching, i.e., $\varepsilon=1, \varpi=$ 0 , the above reduces to $\widetilde{\mathcal{P}}_{\mathrm{SCD}}^{\mathrm{s}, \mathrm{mm}} \approx \mathcal{P}_{j, \mathrm{SCD}}^{\mathrm{s}, \mathrm{mm}}(1) \frac{M^{1-\gamma}-1}{J^{1-\gamma}-1}$.

The problem (23) can be approximated as

$$
\begin{array}{ll}
\max _{\varepsilon, \varpi} & \widetilde{\mathcal{P}}_{\mathrm{SCD}}^{\mathrm{s}, \mathrm{mm}} \\
\text { s.t. } & \mathrm{C} 1-\mathrm{C} 3 .
\end{array}
$$

Since $\varepsilon$ and $\varpi$ are coupled in the objective function of (26), we use a decomposition approach to solve this problem. Because 
$M^{1-\gamma}$ is always positive, given $\varpi$, the optimal $\varepsilon$ is obtained by solving the following equivalent sub-problem:

$$
\max _{0 \leqslant \varepsilon \leqslant 1} \frac{\left(\ell_{o}^{\mathrm{s}, \mathrm{mm}}-1\right) \varepsilon^{1-\gamma}+\left(\varepsilon+\frac{(1-\varepsilon)}{\varpi}\right)^{1-\gamma}-\ell_{o}^{\mathrm{s}, \mathrm{mm}}}{J^{1-\gamma}-1}
$$

where $\ell_{o}^{\mathrm{s}}=\frac{\mathcal{P}_{j, \mathrm{SCD}}^{\mathrm{s}}(1)}{\mathcal{P}_{j, \mathrm{SCD}}^{\mathrm{s}}(\varpi)}, \ell_{o}^{\mathrm{mm}}=\frac{\left(\mathcal{P}_{j, \mathrm{SCD}}^{\mathrm{mm}, \mathrm{L}}(1)+\mathcal{P}_{j, \mathrm{SCD}}^{\mathrm{mm}, \mathrm{N}}(1)\right)}{\left(\mathcal{P}_{j, \mathrm{SCD}}^{\mathrm{m} L}(\varpi)+\mathcal{P}_{j, \mathrm{SCD}}^{\mathrm{m}, \mathrm{N}}(\varpi)\right)}$ are independent of $\varepsilon$. Thus, we have the following theorem.

Theorem 3: The optimal solution of the problem (27) is given by

$$
\varepsilon^{*}=\min \left(\max \left(\varepsilon_{o}, 0\right), 1\right),
$$

where $\varepsilon_{o}=\left(\left(\left(\frac{\ell_{o}^{\mathrm{s}, \mathrm{mm}}-1}{\varpi^{-1}-1}\right)^{-1 / \gamma}-1\right) \varpi+1\right)^{-1}$.

Proof 3: Let $f_{1}(\varepsilon)$ denote the objective function of the problem (27). We can obtain the first-order and the secondorder derivatives of $f_{1}(\varepsilon)$ with respective to (w.r.t.) $\varepsilon$ as

$$
\begin{gathered}
\frac{\partial f_{1}(\varepsilon)}{\partial \varepsilon}=\frac{(1-\gamma)}{J^{1-\gamma}-1}\left[\frac{\ell_{o}^{\mathrm{s}, \mathrm{mm}}-1}{\varepsilon^{\gamma}}+\left(1-\frac{1}{\varpi}\right) \mathcal{K}^{-\gamma}\right], \text { (29) } \\
\frac{\partial^{2} f_{1}(\varepsilon)}{\partial \varepsilon^{2}}=\frac{-\gamma(1-\gamma)}{J^{1-\gamma}-1}\left[\frac{\ell_{o}^{\mathrm{s}, \mathrm{mm}}-1}{\varepsilon^{\gamma+1}}+\left(1-\frac{1}{\varpi}\right)^{2} \mathcal{K}^{-\gamma-1}\right],
\end{gathered}
$$

respectively, where $\mathcal{K}=\varepsilon+\frac{1-\varepsilon}{\varpi}$. Note that $\ell_{o}^{\mathrm{s}, \mathrm{mm}} \geqslant 1$ and $\frac{1-\gamma}{J^{1-\gamma}-1}>0$, so we get $\frac{\partial^{2} f_{1}(\varepsilon)}{\partial \varepsilon^{2}} \leqslant 0$, which means that $f_{1}(\varepsilon)$ is a concave function w.r.t. $\varepsilon$. By setting $\frac{\partial f_{1}(\varepsilon)}{\partial \varepsilon}$ to zero, we obtain the stationary point $\varepsilon_{o}$.

Note that $0 \leqslant \varpi \leqslant 1$, and $\frac{\ell_{o}^{\mathrm{s}, \mathrm{mm}}-1}{\varpi^{-1}-1} \geqslant 0$, we have $\varepsilon_{o} \geqslant 0$. To obtain the optimal $\varepsilon^{*}$, we need to consider the following cases:

- Case 1: $0 \leqslant \varepsilon_{o}<1$. In this case, the optimal solution of the problem (27) is $\varepsilon^{*}=\varepsilon_{o}$.

- Case 2: $\varepsilon_{o} \geqslant 1$. In this case, $\frac{\partial f_{1}(\varepsilon)}{\partial \varepsilon} \geqslant 0$ for $\varepsilon \in[0,1]$, and thus the optimal solution of the problem (27) is $\varepsilon^{*}=1$.

Based on the above cases, we obtain (28) and complete the proof. For $\varepsilon_{o}$ to be in the range of $[0,1], \varpi$ should satisfy $0 \leqslant \varpi \leqslant \frac{1}{\ell_{0}^{s, \mathrm{mmm}}}$.

Consequently, the problem (26) reduces to the following optimization problem about $\varpi$ only:

$$
\max _{\varepsilon=\varepsilon_{o}(\varpi), 0 \leqslant \varpi \leqslant \frac{1}{\ell_{o}^{s, 1 m m}}} \widetilde{\mathcal{P}}_{\text {SCD }}^{\mathrm{s}, \mathrm{mm}} .
$$

Since the problem (31) is non-convex, the optimal value can be found via one-dimensional search.

\section{RESULTS AND DISCUSSIONS}

In this section, the performance of the proposed caching scheme are evaluated using numerical results. Performance comparison between cache-enabled sub- $6 \mathrm{GHz}$ and mmWave systems is also highlighted. The system parameters are shown in Table I, unless otherwise specified. $1 \mathrm{GHz}$ and $60 \mathrm{GHz}$ are chosen for the sub- $6 \mathrm{GHz}$ and mmWave frequency bands, respectively.
TABLE I

PARAMETER VALues.

\begin{tabular}{c|c}
\hline Parameters & Values \\
\hline \hline Number of Antenna in sub-6 GHz-SBS $\left(N_{\mathrm{S}}\right)$ & 2 \\
\hline Main-lobe Array Gain in mmWave-SBS $\left(G_{\mathrm{mm}}\right)$ & 2 \\
\hline LOS region $\left(D_{\mathrm{L}}\right)$ & $15 \mathrm{~m}$ \\
\hline Transmit power of each sub-6 GHz-SBS $P_{\mathrm{S}}$ & $20 \mathrm{dBm}$ \\
\hline Transmit power of each mmWave-SBS $P_{\mathrm{mm}}$ & $20 \mathrm{dBm}$ \\
\hline SBS's density for sub-6 GHz and $\mathrm{mmWave}$ & $\lambda_{\mathrm{s}}, \lambda_{\mathrm{mm}}=600 / \mathrm{km}^{2}$ \\
\hline Path loss exponent $f_{c}=1 \mathrm{GHz}$ & $\alpha_{\mathrm{s}}=2.5$ \\
\hline Path loss exponent $f_{c}=60 \mathrm{GHz}[17]$ & $\alpha_{\mathrm{L}}=2.25, \alpha_{\mathrm{N}}=3.76$ \\
\hline Bit rate of each file $(\eta / T)$ & $4 \times 10^{5} \mathrm{bit} / \mathrm{s}$ \\
\hline Available bandwidth in sub-6 $\mathrm{GHz}\left(W_{\mathrm{s}}\right)$ & $10 \mathrm{MHz}$ \\
\hline Available bandwidth in mmWave $\left(W_{\mathrm{mm}}\right)$ & $1 \mathrm{GHz}$ \\
\hline SBS cache capacity $(M)$ & 100 \\
\hline Content library size $(J)$ & $10^{4}$ \\
\hline Zipf parameter $\gamma$ & $0 \sim 2$ \\
\hline
\end{tabular}
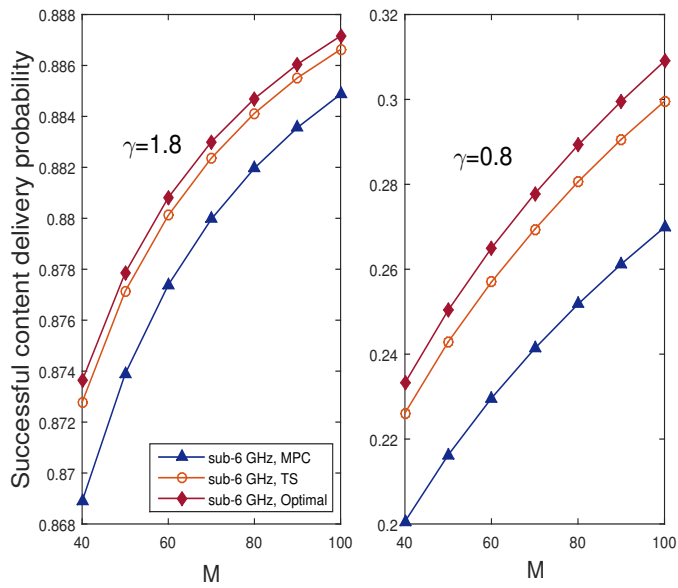

Fig. 1. Successful content delivery probability for sub-6 GHz single antennas.

In Fig. 1, we compare the performance of the proposed content placement scheme with the optimal solution [15] and the MPC scheme in the sub- $6 \mathrm{GHz}$ single-antenna case. Note that in the general multi-antenna setting, the optimal content placement is still unknown. The SCDP with different caching capacity $M$ is shown in Fig. 1. It is observed that the proposed scheme provides close-to-optimal and significantly better performance than the MPC solution, especially when $\gamma$ is small. The MPC solution is the worst caching scheme because it ignores the content diversity which is particular important when the content popularity is more uniform.

Fig. 2 shows the SCDPs comparison of various systems with different caching capacities $M$. It shows that the proposed content placement scheme performs consistently better than MPC, especially for smaller caching capacities. The results also indicate that sub- $6 \mathrm{GHz}$ always has a superior performance compared to the $60 \mathrm{GHz}$ mmWave with the same SBS density.

Finally, we investigate the cache-density tradeoff and its implication on the comparison of sub- $6 \mathrm{GHz}$ and mmWave systems using the proposed content placement scheme. Fig. 3 


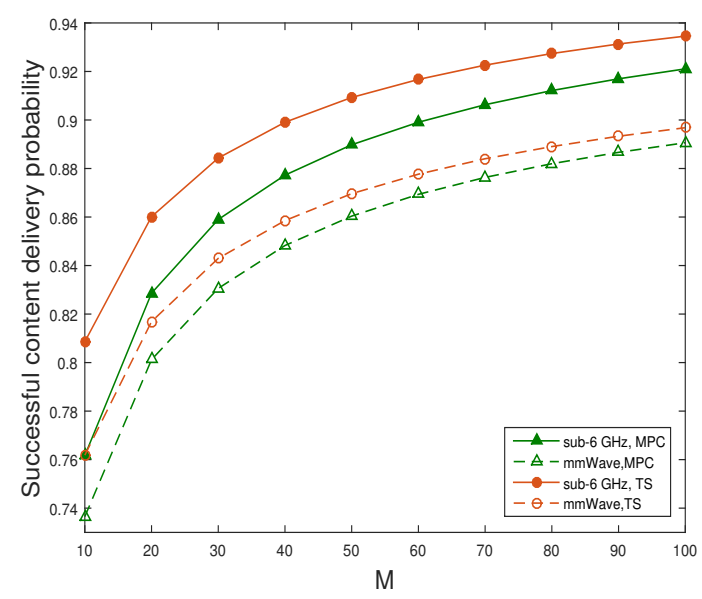

Fig. 2. The impact of $M$ on the successful content delivery probability, $\gamma=$ 1.5 .

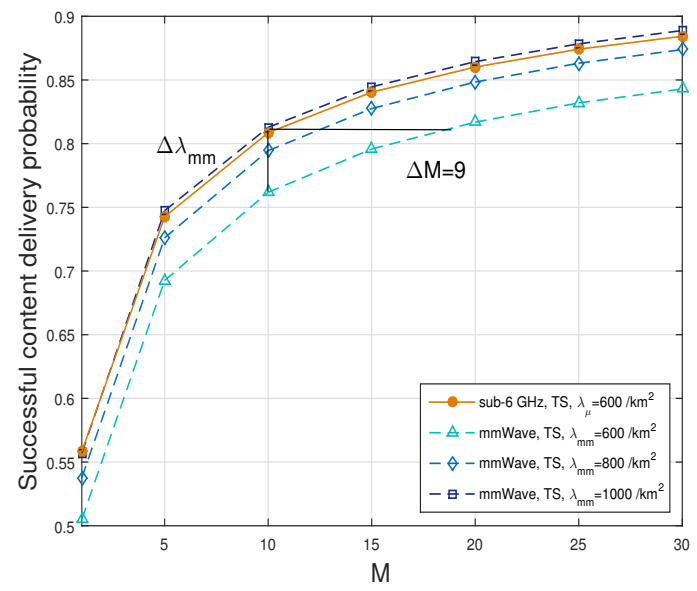

Fig. 3. Cache-density tradeoff, $\gamma=1.5$.

demonstrates the SCDPs with different caching capacity $M$, SBS densities $\lambda_{\mathrm{s}}$ and $\lambda_{\mathrm{mm}}$. It is observed that the sub-6 GHz channel is usually better than the mmWave channel, so with the same SBS density, sub-6 GHz achieves higher SCDP. To achieve performance comparable to that of the sub- $6 \mathrm{GHz}$ system with SBS density of $600 / \mathrm{km}^{2}$, the mmWave needs to deploy SBSs with a much higher density of $1000 / \mathrm{km}^{2}$, but the extra density of $\triangle \lambda_{\mathrm{mm}}=400 / \mathrm{km}^{2}$ is too costly to afford. Fortunately, by increasing the caching capacity from 10 to 19 , the mmWave system can achieve the same SCDP of $82 \%$ as the sub- $6 \mathrm{GHz}$ system while keeping the same density of $600 / \mathrm{km}^{2}$. This result shows great promise of cache-enabled small cell systems because the relatively cheap storage can compensate the expensive infrastructure.

\section{CONCLUSION}

This paper we have investigated the performance of caching in sub-6 GHz and mmWave multi-antenna dense networks to improve the efficiency of content delivery. Using stochastic geometry, we have analyzed the successful content delivery probabilities and demonstrated the impact of various system parameters. We designed a novel caching scheme to maximize the successful content delivery probability with low complexity, and it has been shown to achieve near-optimal performance in the single-antenna case. An important implication of this work is that to reduce the performance gap between the sub$6 \mathrm{GHz}$ and mmWave systems, increasing caching capacity is a low-cost and effective solution compared to the traditional measures such as using more antennas or increasing SBS density. As a promising future direction, to study cooperative caching in a hybrid sub- $6 \mathrm{GHz}$ and mmWave system could further reap the benefits of both systems.

\section{REFERENCES}

[1] C. Fang, F. R. Yu, T. Huang, J. Liu, and Y. Liu, "A survey of energyefficient caching in information-centric networking," IEEE Commun. Mag., vol. 52, no. 11, pp. 122-129, Nov. 2014.

[2] E. G. Larsson, T. L. Marzetta, H. Q. Ngo, and H. Yang, "Antenna Count for Massive MIMO: $1.9 \mathrm{GHz}$ versus $60 \mathrm{GHz}$," arXiv preprint arXiv:1702.06111, Feb. 2017.

[3] T. Bai and R. W. Heath Jr, "Comparing massive MIMO and mmWave massive MIMO," 2016. [Online]. Available: http://users.ece.utexas.edu/ rheath/presentations/2015/ ComparingMassiveMIMOSub6GHzAndMmWaveICC2015Heath.pdf

[4] J. Qiao, Y. He, and X. S. Shen, "Proactive caching for mobile video streaming in millimeter wave 5G networks," IEEE Trans. Wireless Commun., vol. 15, no. 10, pp. 7187 - 7198, Aug. 2016.

[5] Z. Chen, J. Lee, T. Q. Quek, and M. Kountouris, "Cooperative caching and transmission design in cluster-centric small cell networks," arXiv preprint arXiv:1601.00321, 2016.

[6] G. Zheng, H. Suraweera, and I. Krikidis, "Optimization of hybrid cache placement for collaborative relaying," IEEE Commun. Lett., vol. 21, no. 2 , pp. $442-445$, Feb. 2017

[7] D. Malak, M. Al-Shalash, and J. G. Andrews, "Optimizing content caching to maximize the density of successful receptions in device-todevice networking," arXiv preprint arXiv:1608.07857, 2016.

[8] — "Spatially correlated content caching for device-to-device communications," arXiv preprint arXiv:1609.00419, 2016.

[9] B. Blaszczyszyn and A. Giovanidis, "Optimal geographic caching in cellular networks," in Proc., IEEE Int. Conf. Commun. (ICC), London, UK, Sept. 2015, pp. 3358-3363.

[10] T. S. Rappaport, S. Sun, R. Mayzus, H. Zhao, Y. Azar, K. Wang, G. N. Wong, J. K. Schulz, M. Samimi, and F. Gutierrez, "Millimeter wave mobile communications for $5 \mathrm{G}$ cellular: It will work!" IEEE Access, vol. 1, pp. 335-349, May 2013.

[11] T. Bai and R. W. Heath Jr., "Coverage and rate analysis for millimeterwave cellular networks," IEEE Trans. Wireless Commun., vol. 14, no. 2, pp. 1100-1114, Feb. 2015.

[12] J. Park, S. L. Kim, and J. Zander, "Tractable resource management with uplink decoupled millimeter-wave overlay in ultra-dense cellular networks," IEEE Trans. Wireless Commun., vol. 15, no. 6, pp. 43624379, Jun. 2016.

[13] Y. Zhu, L. Wang, K.-K. Wong, and R. W. Heath, "Secure communications in millimeter wave ad hoc networks," IEEE Trans. Wireless Commun., vol. 16, no. 5, pp. 3205-3217, May 2017.

[14] K. Venugopal, M. C. Valenti, and R. W. Heath, "Device-to-device millimeter wave communications: Interference, coverage, rate, and finite topologies," IEEE Trans. Wireless Commun., vol. 15, no. 9, pp. 61756188, Jun. 2016.

[15] J. Wen, K. Huang, S. Yang, and V. O. Li, "Cache-enabled heterogeneous cellular networks: Optimal tier-level content placement," arXiv preprint arXiv:1612.05506, 2016.

[16] M. Taghizadeh, K. Micinski, S. Biswas, C. Ofria, and E. Torng, "Distributed cooperative caching in social wireless networks," IEEE Trans. Mobile Comput., vol. 12, no. 6, pp. 1037-1053, Jun. 2013.

[17] T. S. Rappaport, E. Ben-Dor, J. N. Murdock, and Y. Qiao, "38 GHz and $60 \mathrm{GHz}$ angle-dependent propagation for cellular \& peer-to-peer wireless communications," in Proc., IEEE Int. Conf. Commun. (ICC), Ottawa, Canada, Nov. 2012, pp. 4568-4573. 